\title{
Transovarian Transmission of Blochmannia and Wolbachia Endosymbionts in the Neotropical Weaver Ant Camponotus textor (Hymenoptera, Formicidae)
}

\author{
Manuela Oliveira Ramalho ${ }^{1,2}$ D $\cdot$ Alexsandro Santana Vieira ${ }^{1} \cdot$ Mayara Cristina Pereira ${ }^{1} \cdot$ Corrie Saux Moreau $^{2}$ \\ Odair Correa Bueno ${ }^{1}$
}

Received: 7 December 2017 / Accepted: 19 February 2018 / Published online: 21 February 2018

(c) Springer Science+Business Media, LLC, part of Springer Nature 2018

\begin{abstract}
Camponotus is a hyper-diverse ant genus that is associated with the obligate endosymbiont Blochmannia, and often also with Wolbachia, but morphological studies on the location of these bacteria in the queen's ovaries during oogenesis remain limited. In the present study, we used the Neotropical weaver ant Camponotus textor to characterize the ovary using histology (HE) techniques, and to document the location of Blochmannia and Wolbachia during oogenesis through fluorescence in situ hybridization (FISH). This is the first morphological report of these two bacteria in the same host with polytrophic meroistic ovaries and reveals that Blochmannia is found inside late-stage oocytes and Wolbachia is associated with the nuclei of the nurse cells. Our results provide insights into the developmental sequence of when these bacteria reach the egg, with Blochmannia establishing itself in the egg first, and Wolbachia only reaching the egg shortly before completing egg development. Studies such as this provide understanding about the mechanisms and timing of the establishment of these endosymbionts in the host.
\end{abstract}

Electronic supplementary material The online version of this article (https://doi.org/10.1007/s00284-018-1459-3) contains supplementary material, which is available to authorized users.

Manuela Oliveira Ramalho

manuramalho2010@gmail.com

Alexsandro Santana Vieira

alexsvieira@yahoo.com.br

Mayara Cristina Pereira

mayarac.pereira@yahoo.com.br

Corrie Saux Moreau

cmoreau@ fieldmuseum.org

Odair Correa Bueno

odaircb@rc.unesp.br

1 Departament of Biology e Center for Studies on Social Insects, Biosciense Institute, São Paulo State University (UNESP), Campus Rio Claro, Avenida 24A, 1515, Bela Vista, Rio Claro, SP 13506-900, Brazil

2 Field Museum of Natural History, Department of Science and Education, Integrative Research Center, 1400 South Lake Shore Drive, Chicago, IL 60605, USA

\section{Introduction}

The ant genus Camponotus Mayr, 1861 is one of the most diverse and has a worldwide distribution [2, 6]. They have generalist diets and can nest in cavities in trees, in hollow or rotten twigs, or in the ground $[13,23]$, and some species construct nests in trees with silk produced by larvae, as in the case of Camponotus textor Forel, 1899 [27, 31]. This group of ants is also known to have obligatory symbiotic relationships with bacteria $[10,11,16,39]$ and studies have shown that the main associated taxa are Blochmannia and Wolbachia, representing about 95-98\% of all sequencing reads of Camponotus chromaiodes Bolton, 1995 [7].

These symbiotic bacteria may have positive or negative effects on the host. Blochmannia, for example, is known for its beneficial effects because it provides a number of amino acids to the host, thus it has a nutritional role, especially in the early developmental stages of host life [10, 11, 16, 41]. For many arthropod hosts, Wolbachia is known for its negative effect in manipulating host reproduction, such as parthenogenesis, death of males, feminization, and cytoplasmic incompatibility (CI) [4, 12, 28, 35]. For bedbugs, it may aid in nutrition with vitamin B supplementation [18]. However, its function in ants is not known, especially in the 
sterile workers, who are not able to reproduce $[1,29,30]$. Both bacteria can be transmitted vertically (maternal inheritance), with Blochmannia acting as a primary and obligatory endosymbiont and Wolbachia as secondary and facultative $[1,11,39]$.

A previous study by Ramalho et al. [24] using next-generation sequencing techniques surveyed the bacterial community present across all stages of development of multiple Camponotus colonies. The main bacteria found in the egg and queen were Blochmannia and Wolbachia, reinforcing the idea that the route of acquisition of these endosymbionts occurs through maternal inheritance. Thus, morphological studies of the reproductive organs of Camponotus ant queens may inform the strategies of the establishment of these endosymbiotic bacteria in the host.

The acquisition of endosymbionts in oviparous insects can occur either at the beginning or in the late stages of oogenesis, although there are few studies in Hymenoptera (bees, wasps, and ants) that exhibit polytrophic meroistic ovaries [22]. Insects with polytrophic meroistic ovaries have nurse cells (grouped inside the nurse chamber) and oocytes that alternate along the length of ovariole. This set of nurse chambers (containing the nurse cells) plus the egg chamber (containing the oocytes) is called the ovarian follicle. To investigate transmission of Wolbachia, several studies have been carried out in Drosophila and tsetse flies, which have meroistic telotrophic ovaries, in which the nurse chamber is located basally and the apical region is where the germarium (containing the stem cells) is found $[3,9,14]$. Frydman et al. [14] were able to experimentally add Wolbachia to the Drosophila melanogaster abdomen and to monitor their tissue distribution. They found that only 15 days after the infection, Wolbachia was detected in the germ line and the transmission route was through the somatic stem cell in the germarium.

Blochmannia has been detected using fluorescence in situ hybridization (FISH) during oogenesis of Camponotus floridanus, a species commonly found in the Nearctic region [22]. These authors found that the bacterium was not present in the germarium, nor in the nurse cells, but they were located within the oocyte and believed to have been transferred via follicular cells [22]. As both bacteria, Blochmannia and Wolbachia, were found in large numbers infecting workers of Camponotus textor, an exclusively Neotropical species [25], this presents the opportunity to investigate the localization of these bacteria in the reproductive tract of this species. As the genus Camponotus is very species rich, it is unclear if this observed pattern is conserved across the group.

There are several studies addressing the specific location of these bacteria in host tissues $[1,11,14,22,34,44]$ and a few studies have included insects with telotrophic meroistic ovaries. These studies have found Wolbachia associated with the nucleus of the nurse cells, and it is believed that this bacterium likely reaches the egg through the cytoplasmic bridges that exist between the nurse chamber and the egg chamber [3, 42]. But this bacterium has not been localized in insects that exhibit polytrophic meroistic ovaries. For Blochmannia, in Camponotus floridanus, it is already known that it is present in the young and mature oocytes, via follicular cells in polytrophic meroistic ovaries [22]. This leads to the question of whether other species of Camponotus have the same pattern of distribution for Blochmannia and what is the location of Wolbachia within polytrophic meroistic ovarian tissues during dual infections. In this study, we used fluorescence in situ hybridization (FISH) to document the distributions of Blochmannia and Wolbachia symbionts in detail during the oogenesis of the queen of Camponotus textor and provide a possible developmental mechanism of how these bacteria reach the egg.

\section{Materials and Methods}

The silk nest of Camponotus textor containing adult, immature individuals, breeding and several queens (polygeny), was collected in August 2016 in Araraquara São Paulo, Brazil (Lat. -21.8262, Long. -48.2001). Eight workers were collected and stored in $95 \%$ ethanol for screening of $\mathrm{Wol}$ bachia and Blochmannia infections. Total DNA extraction was performed following the same parameters described by Ramalho et al. [26]. For the confirmation of the presence of these bacteria in the colony, the primers Bloch $16 \mathrm{~S}-462 \mathrm{~F}$ and Bloch 16S-1299R [39] and Wsp81f and Wsp691r [43], for Blochmannia and Wolbachia, respectively, were used to amplify this target region through PCR, following the same parameters of Ramalho et al. [25]. Three C. textor queens from the same colony were dissected in 1X PBS (Fig. 1a). Some ovarioles were submitted to the histological technique Hematoxylin and Eosin (HE) and to the total assembly technique for FISH.

\section{Morphology-Hematoxylin and Eosin}

For this technique, the protocol described by Junqueira and Junqueira [19] was followed. The extracted ovarioles were fixed in $4 \%$ paraformaldehyde (w/v) for $24 \mathrm{~h}(\mathrm{~h})$, and then transferred to buffer solution (Sodium Phosphate $\mathrm{pH}$ 7.4) for $24 \mathrm{~h}$. Subsequently, they were dehydrated in an increasing series of alcohols (50-95\%) over fifteen-minute intervals.

At the end of the dehydration process, the material was transferred to the embedding historesin and held for 5 days. Subsequently, the organs were included in plastic molds containing historesin (Leica Historesin) and polymerizer 3-6 $\mu \mathrm{m}$ thick. The blocks were sectioned on a LEICA RM 2255 microtome. The histological sections were placed on 


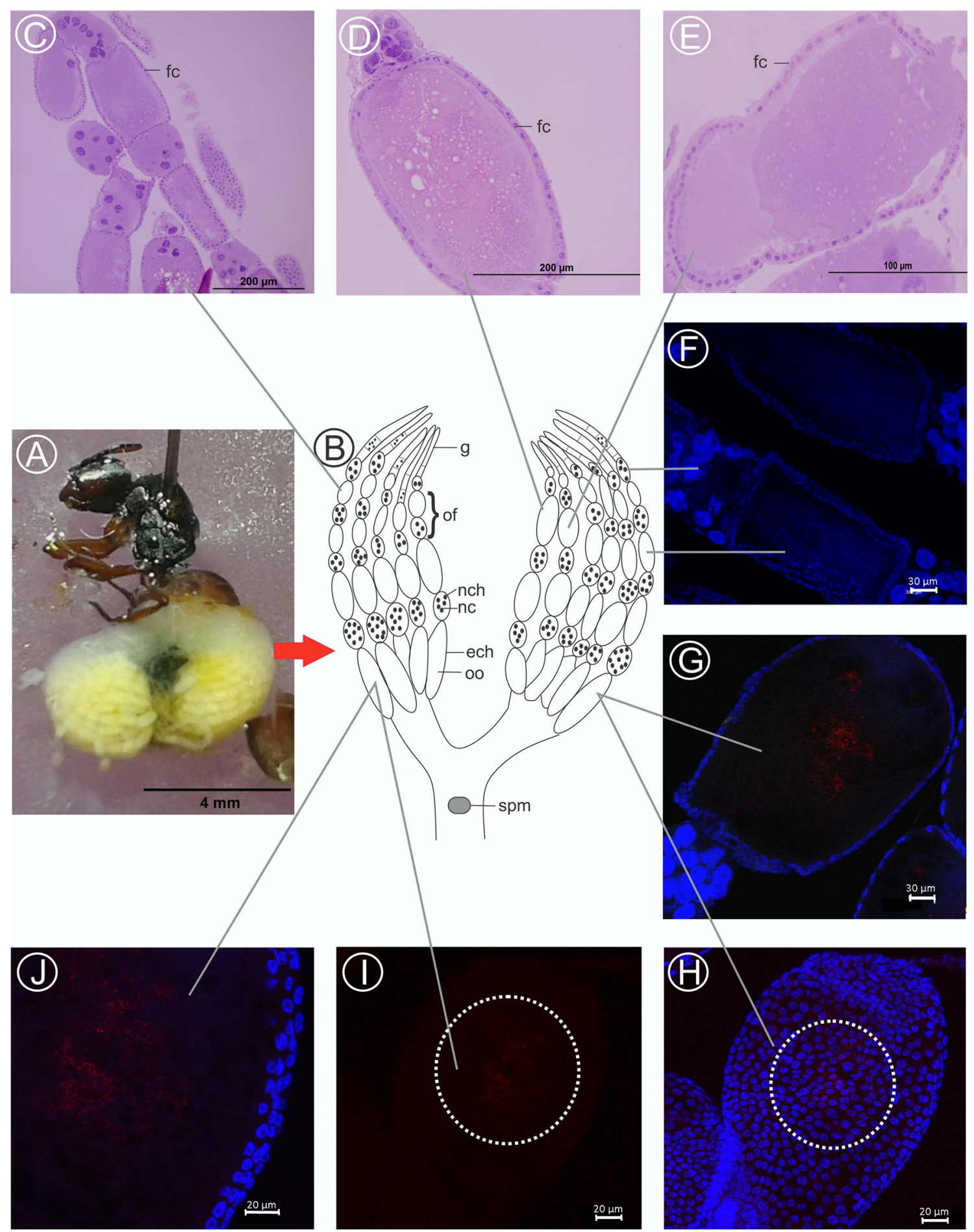

Fig. 1 Schematic representation and photomicrography of the ovaries of a Camponotus textor queen submitted to the histological techniques of HE (Hematoxylin and Eosin) and fluorescence in situ hybridization (FISH) with the presence of Blochmannia in the oocytes. a Right and left ovaries dissected from a $C$. textor queen. b Scheme of the polytrophic meroistic ovary of $C$. textor, with representation of some ovarioles. c Ovarioles with nurse chamber and egg chamber at different stages of development (HE). Note the smaller, younger oocytes and the larger, more mature oocytes. d Ovarian follicle with nurse cells and egg chamber with the late-stage oocyte surrounded by follicular cells (HE). e Mature oocyte surrounded by follicular cells (HE). f Young oocyte and nurse cells without the presence of Blochmannia (FISH). g-j Mature oocytes with Blochmannia marked in red in the central region. Dotted circle highlights Blochmannia. There is no presence of this bacterium in the nurse cells (FISH). $f c$ follicular cells, of ovarian follicle, nch nurse chamber, ech egg chamber, oo oocyte, $n c$ nurse cells, $g$ germarium, spm spermatheca. The process of maturation of the oocyte occurs in the germarium-to-spermatheca direction. Cell nuclei were stained with DAPI and are in blue, and Blochmannia is shown in red (Alexa 647Invitrogen) 
glass slides and hydrated for $1 \mathrm{~min}$ in $\mathrm{dH} 2 \mathrm{O}$ and then stained by Harris hematoxylin for $10 \mathrm{~min}$. After washing for $5 \mathrm{~min}$ with miliQ water, they were stained by aqueous eosin for another $5 \mathrm{~min}$, and again washed in miliQ water. After drying, the slides they were dipped in xylol and then covered with Canada balsam and a cover slip. The permanent slides were examined and photographed under a LEICA DM750 light microscope.

\section{FISH Technique (Fluorescence In Situ Hybridization) and Confocal Microscopy}

The ovarioles were separated and fixed in $4 \%$ (w/v) paraformaldehyde in PBS at room temperature for two hours. Subsequently, they were washed in 50, 70, and $100 \%$ ethanol baths for 3 min each. The material was placed on StarFrost slides (Knittel Glass, Germany), and dried at room temperature. After drying $40 \mu \mathrm{L}$ of hybridization buffer (35\% formamide, $900 \mathrm{mM} \mathrm{NaCl}, 20 \mathrm{mM}$ Tris/ $\mathrm{HCl} \mathrm{pH} \mathrm{7.5,}$ $5 \mathrm{mM}$ EDTA, $0.2 \%$ SDS) preheated together with $2 \mathrm{ng} / \mu \mathrm{L}$ probe (Wolbachia 5' CTAACCCGCCTACGCGCC 3' [1] with Alexa 488-Invitrogen, and Blochmannia 5' CCTATC TGGGTTCATCCAATGGCATAAGGC 3' [11] with Alexa 647-Invitrogen) were added and kept in a humid chamber in the dark at $46{ }^{\circ} \mathrm{C}$ for $2 \mathrm{~h}$. The slides were then washed

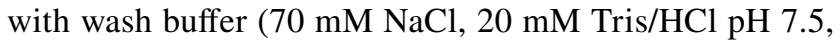
$5 \mathrm{mM}$ EDTA, $0.01 \%$ SDS) and heated again for $30 \mathrm{~min}$ at $48{ }^{\circ} \mathrm{C}$ and also kept in a humid chamber. Subsequently, the excess washing buffer was removed with miliQ water, and it was allowed to dry at room temperature.

The DAPI (Molecular Probes, USA) (1:500) which stains host nuclei blue, and hence possible to infer whether bacteria are intra- or extracellularly present, was placed directly onto the organ for $5 \mathrm{~min}$, and then washed $3 \times$ in miliQ water. Prolong Gold (Thermo Fisher Scientific, USA) was used to mount the slide, which was overlaid with a cover slip and sealed with clear nail polish. For the whole-mount laser, the Leica TCS SP5II confocal microscope was used to obtain the photomicrographs, and Leica TCS SP5II software was used for the confocal analysis using maximum projection. To guarantee the specificity of the analyzed probes, a negative control of the material was performed without any probe used, only with the wash buffer, subjected to wavelength lasers 405, 488, 545, and $647 \mathrm{~nm}$.

\section{Results}

Our PCR-based screening confirmed 100\% Blochmannia and Wolbachia infection in C. textor workers as found in a previous study [25]. The queens of $C$. textor used in the present study showed that the two ovaries developed with more than a hundred ovarioles each (see Fig. 1a). Histological techniques using HE staining showed that the $C$. textor ovary exhibits polytrophic meroistic ovaries, composed of several ovarioles (Fig. 1), with each ovariole composed of the germarium in the distal region (Supplementary File Fig. 1); late or mature oocytes flow into a common oviduct in which the spermatheca is present (see scheme Fig. 1b); oocyte, which can be visualized in the early and late stages of development (Fig. 1c); a chamber of nurse cells with nurse cells (Fig. 1d); and a chamber of eggs surrounded by follicular cells with simple cubic epithelium (Fig. 1e).

Additionally, the probe-based technique, Fluorescent in situ hybridization (FISH), permitted detailed visualization of ovarioles of Camponotus textor queens infected with Wolbachia and Blochmannia. During the analysis of the apical region, which is where the germarium (stem cells) of Camponotus queens (Supplementary File Fig. 1) reside, we did not detect the presence of Blochmannia or even of Wolbachia (Supplementary File Fig. 1). Also, even when the stem cells begin to differentiate in the egg chamber with the oocyte and follicular cells, and the nurse chamber with nurse cells (Figs. 1f, 2a), the presence of Blochmannia and Wolbachia could not be detected. Later, with the advancement of oocyte development, which is correlated with a decrease of the nurse cells (since there are nutrient passages from this cell to the oocyte), the Blochmannia and Wolbachia markings began to appear, but each in a different location: Blochmannia is always found inside the oocyte in a central region (Fig. 1g-J), and Wolbachia is always around the nuclei of the nurse cells (Fig. 2b, c, d, f, g, h). In our results, we did not find any polarization of Blochmannia, that is, it did not appear displaced in any specific region of the oocyte during oocyte development.

\section{Discussion}

Endosymbionts that are transmitted maternally may use different strategies to establish themselves in the oocyte. For example, in Marchalina hellenica (Insecta, Hemiptera), which has a meroistic telotrophic ovary, the infection already appears in the germarium cell, and consequently both the oocytes and the nurse cells have endosymbionts [36]. In the ant, Cardiocondyla obscurior Wheeler, 1929, the Westeberhardia endosymbiont is found in the nurse cells and is only transmitted to the oocytes in the final stages of their development [20].

To better understand how Wolbachia and Blochmannia, two bacteria commonly associated with Camponotus, are maternally transferred [24], it is important to include detailed studies of queen ovarioles using the FISH technique. Here, we identified when and where these bacteria are located in the oogenesis process and below we provide a possible developmental mechanism of how these bacteria 


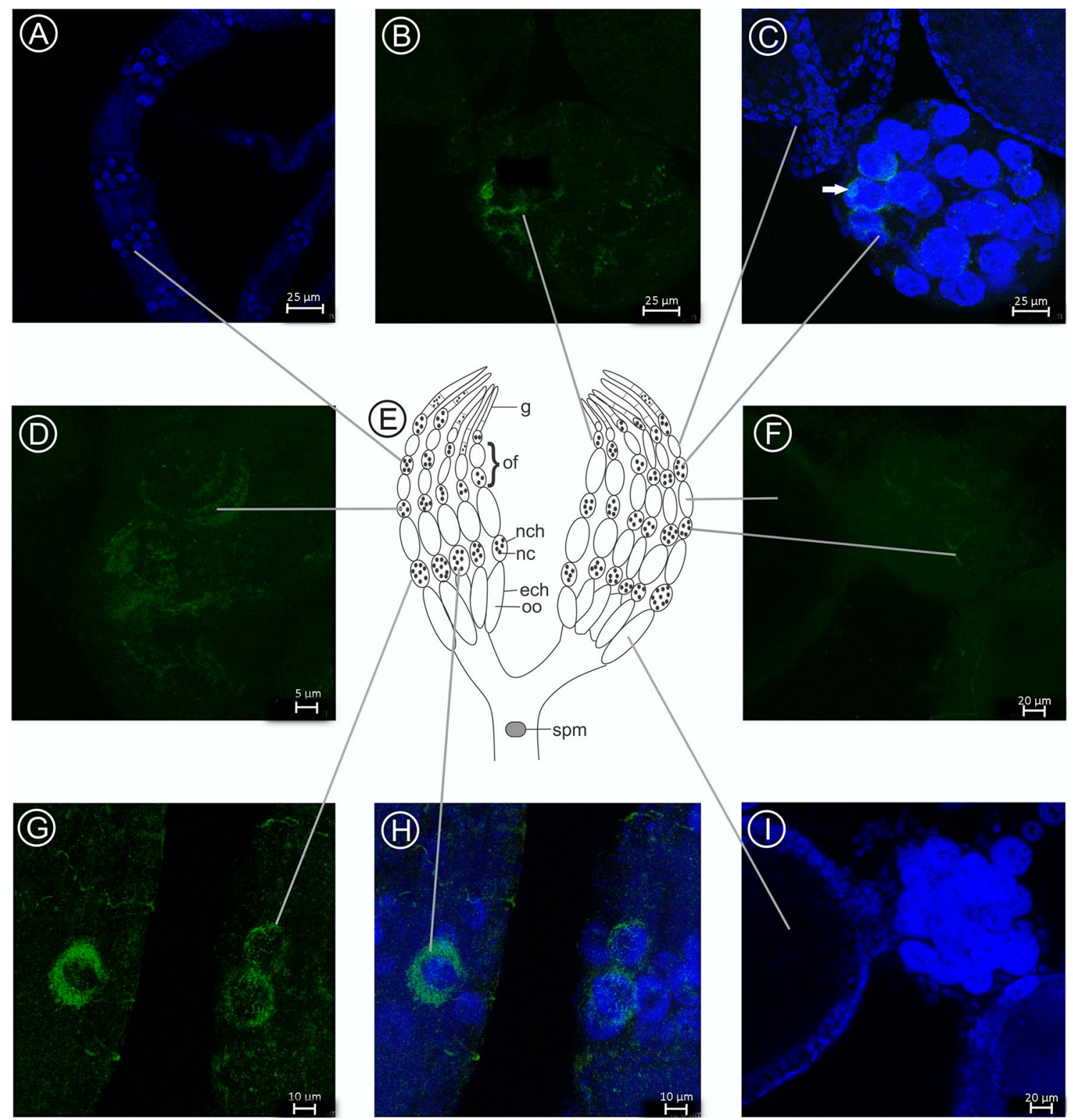

Fig. 2 Schematic representation and photomicrography of the ovaries of Camponotus textor submitted to fluorescence in situ hybridization (FISH) with the presence of Wolbachia in the nurse cells. a Young oocytes and nurse cells without the presence of Wolbachia. b, c, d, $\mathbf{f}, \mathbf{g}, \mathbf{h}$ Presence of Wolbachia around the nucleus of the nurse cells. Arrow highlights Wolbachia around the nucleus. e Scheme of the polytrophic meroistic ovary of $C$. textor, with representation of some ovarioles. i Note that there is no presence of Wolbachia within the oocytes. of ovarian follicle, nch nurse chamber, ech egg chamber, oo oocyte, $n c$ nurse cells, $g$ germarium, spm spermatheca. The process of maturation of the oocyte occurs in the germarium-to-spermatheca direction. Cell nuclei were stained with DAPI and are in blue, and Wolbachia is shown in green (Alexa 488 Invitrogen)

\section{Blochmannia}

Our results documented that Blochmannia is already present in the developing oocytes. This could be explained at this stage of development as Blochmannia is already present in large quantities, permitting detection with these methods. In Camponotus floridanus, Kupper et al. [22] found that 
Blochmannia infection is more prevalent at the beginning of oocyte development, exclusively in the follicular cells and not in the germarium. Our results corroborate data from Camponotus floridanus (Buckley, 1866) [22], as we found no signal of Blochmannia infection in the C. textor germarium region of the ovary. This may suggest that the infection by this bacterium occurs later in development. Some studies of Camponotus have been able to detect infection in young oocytes [5, 8, 22], while others are only able to detect Blochmannia in mature oocytes [32,33] and present study. But Blochmannia, whether in the young or mature oocyte, may access the oocyte via follicular cells $[5,8]$, as the nurse cells do not have this endosymbiont [22], corroborating our results.

Additionally, Kupper et al. [22] were able to visualize the displacement, in a more advanced stage of ovarian development, of Blochmannia to the posterior pole of the oocyte. According to the authors, this may be explained by the high quantity of yolk present in the late oocyte, moving Blochmannia to this region. In our observations, Blochmannia always appears in the central region, and never concentrated towards any of the poles. This migration of the bacterium towards one of the poles may still occur because our oocytes had not yet reached this later developmental stage.

\section{Wolbachia}

Our current understanding of the mechanisms involved in Wolbachia localization is limited [14, 17, 37]. Our results showed that Wolbachia is always present inside the nurse chamber, around the nuclei of the nurse cells, intracellularly. This provides additional evidence for the vertical transference of these bacteria [25]. It must then be passed on to the oocyte at a later developmental stage. We know that this bacterium has been found in the eggs of Camponotus even though it is in low quantities [24].

At the beginning of oogenesis, Wolbachia was not found in the germarium. This suggests that this bacterium must have an alternative mechanism to ensure that it arrives in the nurser chamber later to ensure its vertical transference. Other studies performed with Chalcidoidea wasps and Glossina tsetse flies have also found this same distribution pattern of Wolbachia associated with nurse cells, and being transferred only in the late stages of oogenesis or even in young embryos [3, 42]. Zchori-Fein and collaborators [42] have been able to demonstrate that the bacterium is passed to the oocyte at later stages, via nurse cells, through cytoplasmic bridges. Our data from Wolbachia is the first study to account for meroistic politrophic ovaries in the ant, Camponotus textor, and suggests that the same mechanism happens, since we only observe Wolbachia around the nucleus of the nurse cells.
For Wolbachia to be passed vertically to the progeny, it seems reasonable that it is present in the stem cells of the germarium. The low amount of Wolbachia in these stem cells could result in the loss of infection that has been observed by Werren [40]. However, some studies have demonstrated that there are different strategies and pathways to ensure that this bacterium is passed to the next generation $[14,15]$. Therefore, although Wolbachia appears to pass into the oocyte via nurse cells shortly before completing development, another strategy could be for different strains of Wolbachia to concentrate at different locations in the oocyte, in order to ensure greater chances of success in the passage to the progeny, as has been observed for Drosophila [21, 38]. The results presented here support the hypothesis of Wolbachia being passed in the late stages of oocyte development for $C$. textor.

\section{Conclusions}

Our study is the first to use histology techniques and fluorencence in situ hybridization to visualize the two main bacteria occurring together in the same host, which likely exhibit different infection strategies, of Camponotus during oogenesis of a polytrophic meroistic ovary. From our findings, we demonstrated that Blochmannia first appears in the oocyte, with Wolbachia only present in the final stages, before the oocyte completes development. Our results corroborate the idea that Blochmannia is transferred into the oocyte via follicular cells, and Wolbachia passes laterally straight from the nurse cells to the oocyte through the cytoplasmic connections between the egg chamber and the nurse chamber. Understanding more about the mechanisms of ovarian transmission of these endosymbionts may reveal more about their adaptations that permit the failure or success of Blochmannia and Wolbachia to colonize new hosts.

Acknowledgements We thank Priscila Cintra Socolowski and Gerson Mello Souza for their help with the techniques used in the present study. M.O.R. thanks CAPES Foundation (process no. 007343/201400) the Ministry of Education of Brazil, (Brasília DF 70.040-020) for their financial support. A.S.V. thanks Conselho Nacional de Desenvolvimento Científico e Tecnológico, grants No. 157837/2015-7. M.C.P. thanks CAPES Foundation. C.S.M. acknowledges the Field Dreams program of The Women's Board of The Field Museum for financial support. O.C.B. thanks Conselho Nacional de Desenvolvimento Científico e Tecnológico.

\section{Compliance with Ethical Standards}

Conflict of interest The authors declare that they have no conflict of interest. 


\section{References}

1. Andersen SB, Boye M, Nash DR, Boomsma JJ (2012) Dynamic Wolbachia prevalence in Acromyrmex leaf-cutting ants: potential for a nutritional symbiosis. J Evol Biol 25:1340-1350. https ://doi.org/10.1111/j.1420-9101.2012.02521.x

2. AntWeb. https://www.antweb.org. Accessed 24 Feb 2017

3. Balmand S, Lohs C, Aksoy S, Heddi A (2013) Tissue distribution and transmission routes for the tsetse fly endosymbionts. J Invertebr Pathol 112:S116-S122. https://doi.org/10.1016/j. jip.2012.04.002

4. Barr AR (1980) Cytoplasmic incompatibility in natural populations of a mosquito, Culex pipiens L. Nature 283:71-72. https ://doi.org/10.1038/283071a0

5. Blochmann F (1882) Über das Vorkommen bakterien€ahnlicher Gebilde in den Geweben und Eiern verschiedener Insekten. Zbl Bakt 11:234-240

6. Bolton B (2016) An online catalog of the ants of the world. http://www.antcat.org/. Accessed 20 Oct 2016

7. Brown BP, Wernegreen JJ (2016) Deep divergence and rapid evolutionary rates in gut-associated Acetobacteraceae of ants. BMC Microbiol doi. https://doi.org/10.1186/s1286 6-016-0721-8

8. Buchner P (1918) Vergleichende Eistudien 1. Die akzessorischen Kerne des Hymenoptereneies. Arch für Mikroskopische Anat 91:1-202. https://doi.org/10.1007/BF02978932

9. Casper-Lindley C, Kimura S, Saxton DS et al (2011) Rapid fluorescence-based screening for Wolbachia endosymbionts in Drosophila germ line and somatic tissues. Appl Environ Microbiol 77:4788-4794. https://doi.org/10.1128/AEM.00215-11

10. Degnan PH, Lazarus AB, Wernegreen JJ (2005) Genome sequence of Blochmannia pennsylvanicus indicates parallel evolutionary trends among bacterial mutualists of insects. Genome Res 15:1023-1033. https://doi.org/10.1101/gr.3771305

11. Feldhaar H, Straka J, Krischke M et al (2007) Nutritional upgrading for omnivorous carpenter ants by the endosymbiont Blochmannia. BMC Biol 5:48. https://doi. org/10.1186/1741-7007-5-48

12. Fenn K, Blaxter M (2004) Are filarial nematode Wolbachia obligate mutualist symbionts? Trends Ecol Evol 19:163-166. https ://doi.org/10.1016/j.tree.2004.01.002

13. Fernandes TT, da Silva RR, de Souza DR et al (2012) Undecomposed twigs in the leaf litter as nest-building resources for ants (Hymenoptera: Formicidae) in areas of the Atlantic forest in the southeastern region of Brazil. Psyche A J Entomol 2012:1-8. https://doi.org/10.1155/2012/89647

14. Frydman HM, Li JM, Robson DN, Wieschaus E (2006) Somatic stem cell niche tropism in Wolbachia. Nature 441:509-512. https://doi.org/10.1038/nature04756

15. Genty L-M, Bouchon D, Raimond M et al (2014) Wolbachia infect ovaries in the course of their maturation: last minute passengers and priority travellers? PLoS ONE 9:e94577. https:// doi.org/10.1371/journal.pone.0094577

16. Gil R, Silva FJ, Zientz E et al (2003) The genome sequence of Blochmannia floridanus: comparative analysis of reduced genomes. Proc Natl Acad Sci USA 100:9388-9393. https://doi. org/10.1073/pnas. 1533499100

17. He L, Wang X, Montell DJ (2011) Shining light on Drosophila oogenesis: live imaging of egg development. Curr Opin Genet Dev 21:612-619. https://doi.org/10.1016/j.gde.2011.08.011

18. Hosokawa T, Koga R, Kikuchi Y et al (2010) Wolbachia as a bacteriocyte-associated nutritional mutualist. Proc Natl Acad Sci USA 107:769-774. https://doi.org/10.1073/pnas.0911476107

19. Junqueira LCU, Junqueira LMMS. (1983) Técnicas básicas de citologia e histologia. Santos, São Paulo
20. Klein A, Schrader L, Gil R et al (2016) A novel intracellular mutualistic bacterium in the invasive ant Cardiocondyla obscurior. ISME J 10:376-388. https://doi.org/10.1038/ismej .2015 .119

21. Kose H, Karr TL (1995) Organization of Wolbachia pipientis in the Drosophila fertilized egg and embryo revealed by an antiWolbachia monoclonal antibody. Mech Dev 51:275-288. https:// doi.org/10.1016/0925-4773(95)00372-X

22. Kupper M, Stigloher C, Feldhaar H, Gross R (2016) Distribution of the obligate endosymbiont Blochmannia floridanus and expression analysis of putative immune genes in ovaries of the carpenter ant Camponotus floridanus. Arthropod Struct Dev 45:475-487. https://doi.org/10.1016/j.asd.2016.09.004

23. Matta LSFDM., Santina De M, Morini MSC, Hilsdorf AWS (2013) Genetic relationship among Camponotus rufipes Fabricius (Hymenoptera:Formicidae) nests by RAPD molecular markers. Acta Sci Biol Sci Mar 35:89-92. https://doi.org/10.4025/actas cibiolsci.v35i1.10913

24. Ramalho MO, Bueno OC, Moreau CS (2017) Species-specific signatures of the microbiome from Camponotus and Colobopsis ants across developmental stages. PLoS ONE 12:e0187461. https ://doi.org/10.1371/journal.pone.0187461

25. Ramalho MO, Martins C, Silva LMR et al (2017) Intracellular symbiotic bacteria of Camponotus textor, Forel (Hymenoptera, Formicidae). Curr Microbiol. https://doi.org/10.1007/s0028 4-017-1201-6

26. Ramalho MO, Martins C, Silva LMR et al (2016) Molecular profile of the brazilian weaver ant Camponotus textor Forel (Hymenoptera, Formicidae). Neotrop Entomol 45:463-470. https://doi. org/10.1007/s13744-016-0392-z

27. Ramalho MO, Santos RM, Fernandes TT et al (2016) Cytochrome c oxidase I DNA sequence of Camponotus ants with different nesting strategies is a tool for distinguishing between morphologically similar species. Genetica 144:375-383. https://doi.org/10.1007/ s10709-016-9906-1

28. Rousset F, Raymond M (1991) Cytoplasmic incompatibility in insects: why sterilize females? Trends Ecol Evol 6:54-57. https ://doi.org/10.1016/0169-5347(91)90123-F

29. Russell JA (2012) The ants (Hymenoptera: Formicidae) are unique and enigmatic hosts of prevalent Wolbachia (Alphaproteobacteria) symbionts. Myrmecol News Myrmecol News 16:7-23

30. Russell JA, Sanders JG, Moreau CS (2017) Hotspots for symbiosis: function, evolution, and specificity of ant-microbe associations from trunk to tips of the ant phylogeny (Hymenoptera: Formicidae). Myrmecol News 24:43-69

31. Santos JC, Del-Claro K (2009) Ecology and behaviour of the weaver ant Camponotus (Myrmobrachys) senex. J Nat Hist 43:1423-1435. https://doi.org/10.1080/00222930902903236

32. Sauer C, Dudaczek D, Hölldobler B, Gross R (2002) Tissue localization of the endosymbiotic bacterium Candidatus Blochmannia floridanus in adults and larvae of the carpenter ant Camponotus floridanus. Appl Environ Microbiol 68:4187-4193. https://doi. org/10.1128/AEM.68.9.4187-4193.2002

33. Schröder D, Deppisch H, Obermayer M et al (1996) Intracellular endosymbiotic bacteria of Camponotus species (carpenter ants): systematics, evolution and ultrastructural characterization. Mol Microbiol 21:479-489. https://doi.org/10.1111/j.1365-2958.1996. tb02557.x

34. Stoll S, Feldhaar H, Fraunholz MJ, Gross R (2010) Bacteriocyte dynamics during development of a holometabolous insect, the carpenter ant Camponotus floridanus. BMC Microbiol 10:308. https://doi.org/10.1186/1471-2180-10-308

35. Stouthamer R, Breeuwer JAJ, Hurst GDD (1999) Wolbachia Pipientis: microbial manipulator of arthropod reproduction. Annu Rev Microbiol 53:71-102. https://doi.org/10.1146/annurev.micro .53 .1 .71 
36. Szklarzewicz T, Kalandyk-Kolodziejczyk M, Kot M, Michalik A (2013) Ovary structure and transovarial transmission of endosymbiotic microorganisms in Marchalina hellenica (Insecta, Hemiptera, Coccomorpha: Marchalinidae). Acta Zool 94:184-192. https ://doi.org/10.1111/j.1463-6395.2011.00538.x

37. Toomey ME, Panaram K, Fast EM et al (2013) Evolutionarily conserved Wolbachia-encoded factors control pattern of stemcell niche tropism in Drosophila ovaries and favor infection. Proc Natl Acad Sci USA 110:10788-10793. https://doi.org/10.1073/ pnas. 1301524110

38. Veneti Z, Clark ME, Karr TL et al (2004) Heads or tails: hostparasite interactions in the Drosophila-Wolbachia system. Appl Environ Microbiol 70:5366-5372. https://doi.org/10.1128/ AEM.70.9.5366-5372.2004

39. Wernegreen JJ, Kauppinen SN, Brady SG, Ward PS (2009) One nutritional symbiosis begat another: phylogenetic evidence that the ant tribe Camponotini acquired Blochmannia by tending sap-feeding insects. BMC Evol Biol 9:292. https://doi. org/10.1186/1471-2148-9-292

40. Werren JH (2005) Heritable microorganisms and reproductive parasitism. In: Sapp J (ed) Microbial phylogeny and evolution: concepts and controversies. Oxford University Press, Oxford, pp 290-315

41. Wolschin F, Hölldobler B, Gross R, Zientz E (2004) Replication of the endosymbiotic bacterium Blochmannia floridanus is correlated with the developmental and reproductive stages of its ant host. Appl Environ Microbiol 70:4096-4102. https://doi. org/10.1128/AEM.70.7.4096-4102.2004

42. Zchori-Fein E, Roush RT, Rosen D (1998) Distribution of parthenogenesis-inducing symbionts in ovaries and eggs of Aphytis (Hymenoptera: Aphelinidae). Curr Microbiol 36:1-8. https://doi. org/10.1007/s002849900270

43. Zhou W, Rousset F, O'Neill S (1998) Phylogeny and PCR-based classification of Wolbachia strains using wsp gene sequences. Proc R Soc London B 265:509-515

44. Zhukova M, Sapountzis P, Schiøtt M, Boomsma JJ (2017) Diversity and transmission of gut bacteria in Atta and Acromyrmex leafcutting ants during development. Front Microbiol 8:1942. https:// doi.org/10.3389/fmicb.2017.01942 